\title{
Comparison between whitened generalized cross correlation and adaptive filter for time delay estimation with scattered arrays for passive positioning of moving targets in Baltic Sea shallow waters
}

\author{
V. Zetterberg \\ Amlab Elektronik AB \\ Nettovaegen 11, Jaerfaella, Sweden \\ Phone: +46 704411220 Email:viktoria.zetterberg@amlab.se \\ M. I. Pettersson \\ Department of Signal Processing, School of Engineering, Blekinge Institute of Technology \\ Soft Center, Ronneby, Sweden \\ Phone: +46457385728 Email:mtp@bth.se \\ I. Claesson \\ Department of Signal Processing, School of Engineering, Blekinge Institute of Technology \\ Soft Center, Ronneby, Sweden \\ Phone: +46457385717 Email:ingvar.claesson@bth.se
}

\begin{abstract}
Results from an acoustic measurement campaign in shallow waters (at 22 meters depth) are given. Eight hydrophones, placed at the seabed, were used simultaneously to investigate how different distances between hydrophones affect the Time Delay Estimation (TDE). In order to position targets two TDE methods were applied. The used methods were the Generalized Cross Correlation (GCC) function weighted by the Phase Transform (PHAT) and an adaptive Normalized Least Mean Square (NLMS) filter estimating the TDE between pairs of sensors. The two methods gave approximately the same result, except for the largest baseline where the GCC PHAT performed better.

In the study we found that increasing separation distance decrease the bandwidth of coherence between the signals, however the study also indicated that scattered arrays with large baselines has better positioning capability than those with small baselines.
\end{abstract}

\section{INTRODUCTION}

The benefit of using larger arrays is coverage of larger areas using the same amount of sensors and giving higher positioning accuracy assuming equal coherence. In this paper we try to analyze how much the result is affected by larger separation distances of hydrophones by using real measurement data. The commonly used way for localization of a sound source is by using linear arrays with small baselines. Little work seems to be conducted about widely and randomly distributed sensors. Xerri, Cavassilas and Burloz [1] have though positioned moving broad band sources with sensors distributed about $400 \mathrm{~m}$ in average from each other at dept $250 \mathrm{~m}$.

Time Delay Estimation (TDE) between at least two different pairs of spatially separated sensors is a robust and computationally practical way [2] that leads to the positioning of a sound source. Since the estimation is the first step, the quality of the estimation affects the rest of the result [3]. By using real measurement data, a result of TDE by two different methods for different baselines is presented. The purpose is to investigate how the TDE is affected by the size of the baseline in shallow waters.
Adaptive filters for TDE eliminating multipath have been studied since the beginning of the 1980's, results from simulated data can be found in e.g. [4,5]. Results from measurements of underwater data are though hard to find. We here present results from The Normalized Least Mean Square (NLMS) adaptive filter [6] applied to underwater measurement data and compared to results from the Generalized Cross Correlation function (GCC) [7], weighted by the Phase Transform (PHAT) [8]. A similar study to this has been done by [9] where simulated data were used.

\section{MEASUREMENT}

The measurement site was along the Swedish coastline of the low salinity Baltic Sea at a depth of 22 meters. Eight hydrophones formed three equilateral triangles with three different bases, $d$ equal to $0.5,9$ and 30 meters. The hydrophones were omni-directional and placed at 0.5 meters above the seabed. A sampling frequency of $48 \mathrm{kHz}$ with a receiver dynamic range of 16 bits was used.

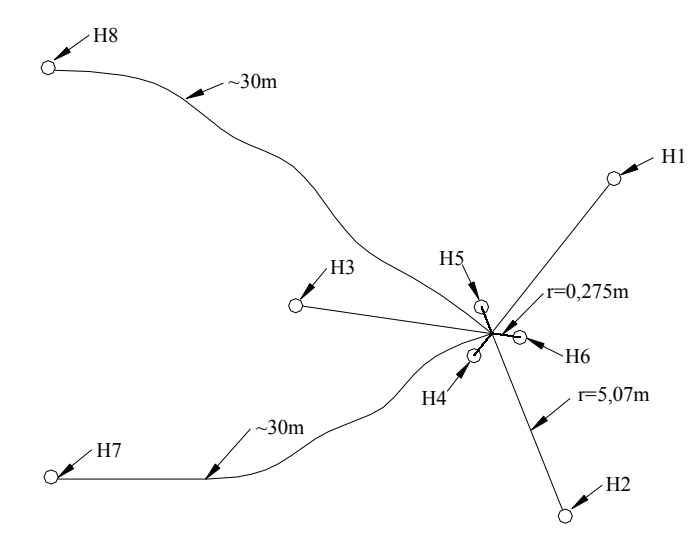

Fig. 1 During the experiment, eight hydrophones was placed at the seabed. Hydrophones 4-5-6 form an equilateral triangle with a baseline of $0.5 \mathrm{~m}$. Hydrophones 1-2-3 form the equilateral triangle with the baseline of $9 \mathrm{~m}$. Hydrophones 3-7-8 form a triangle with a baseline of $30 \mathrm{~m}$. 


\section{GENERALIZED CROSS CORRELATION FUNCTION IN COMPARISON WITH AN ADAPTIVE FILTER}

When a sound source is emitting sound in a field, two signals $\mathrm{x}_{\mathrm{i}}(\mathrm{t})$ and $\mathrm{x}_{\mathrm{j}}(\mathrm{t})$, received at two spatially separated hydrophones, $i$ and $j$, can mathematically be modeled as

$$
\begin{aligned}
& x_{i}(t)=s(t)+n_{1}(t) \\
& x_{j}(t)=s(t-D)+n_{2}(t)
\end{aligned}
$$

where $s(t)$ is the signal (direct path) from the source, $n_{1}(t)$ and $\mathrm{n}_{2}(\mathrm{t})$ are assumed to be mutually uncorrelated noises and $\mathrm{D}$ is time delay. This model does not consider multipath or Doppler effects.

The GCC function [7] weighted by a weighting function, $\mathrm{W}(f)$, is the first method used in this study. The GCC function is given by

$$
\mathrm{R}_{x_{i} x_{j}}(\tau)=\int_{-\infty}^{\infty} \mathrm{W}(f) \mathrm{G}_{x_{i} x_{j}}(f) \mathrm{e}^{\mathrm{j} 2 \pi f \tau} d f
$$

where $\mathrm{G}_{x_{i} x_{j}}$ is the cross spectral density between the signals $x_{i}$ and $x_{j}$. The main purpose of the weighting function (transformation) is here to whiten the cross spectra in order to de-emphasize dominant frequency components. The Phase Transform (PHAT) [8] whitens the cross spectra totally and de-emphasize multipath [2]. PHAT weighting is given from

$$
\mathrm{W}(f)=1 /\left|\mathrm{G}_{x_{i} x_{j}}(f)\right|
$$

The second method is an adaptive FIR-filter identifying TDE between pairs of sensors. Different algorithms for adaptation can be used and in this study we have used the Normalized Least Mean Square (NLMS).

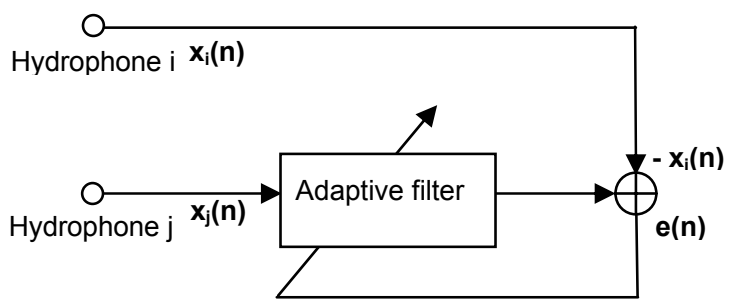

Fig. 2 Adaptive NLMS-filter for TDE.

The adapted filter corresponds to the time delay between the signals $\mathrm{x}_{\mathrm{i}}$ and $\mathrm{x}_{\mathrm{j}}$ from hydrophone $\mathrm{i}$ and $\mathrm{j}$ respectively.

The NLMS algorithm [6] is a gradient searching method that tries to minimize the least mean square difference between the desired signal and the filtered signal. The step size in the searching algorithm must vary if the signals are non-stationary. The NLMS adjusts the step-size according to the energy of the signal. The NLMS is defined by

$$
b_{n+1}=b_{n}+\beta\left(x_{i}(n) /\left\|x_{i}(n)\right\|^{2}\right) e(n)
$$

where $b$ is the filter coefficients of the adaptive filter in Fig. $2, \beta$ is the step size and $\left\|x_{i}(n)\right\|$ is the Euclidean norm [6] of the vector $x_{i}(n)$. Since we use the calculated filter coefficients of the previous time frame as a start value to the next time frame, the step size and signal energy $\left(\left\|x_{i}(n)\right\|^{2}\right)$ affects the relationship between new and old time frame.

One way of improving the filter coefficients for a time frame, after adaptation, is to recalculate the coefficients using the samples of the same time frame again. We call this looping, and this has been used to squeeze out the best result obtainable for NLMS. This is necessary when the integrating time must be held short (due to non- stationary signals) at the same time as we use a low sampling frequency. This is also a way to lessen the affect of the previous time frame.

The advantage of using adaptive filters is that the information in the previous calculated time frame can be used and therefore the time estimation can be updated more often. When the GCC function is used the integration time should be as long as possible without the signal becoming non-stationary due to the displacement of the target. The speed of the target therefore defines the limit of integration time for the GCC.

\section{RESULT}

Results from the two methods applied on one target for the three different baselines are shown below. The position of the target was tracked by a land based laser system. The track is shown in figure 3 . The target is approximately $10 \mathrm{x}$ 20 meter. The angle of each sensor pair in relation to the trajectory is approximately the same, using $\mathrm{H} 8$ to $\mathrm{H} 1, \mathrm{H} 3$ to $\mathrm{H} 2$ and $\mathrm{H} 5$ to H6. These pairs form the three different baselines.

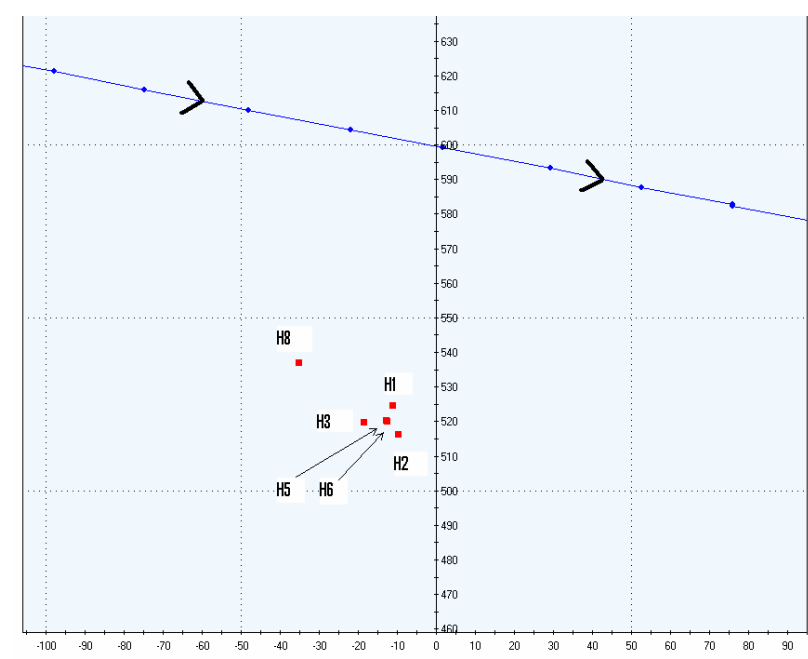

Fig. 3 Trajectory of the target past the hydrophones H8-H1, H3-H2 and $\mathrm{H} 5-\mathrm{H} 6$, where the scale is in meters.

\section{A. Coherence}

The Magnitude Square Coherence (MSC) [10] between the different pairs is shown in Fig. 4. Notice the different scales of the y-axis. We can clearly see that the coherence decreases with increasing size of baseline, $d$. At $d=30 \mathrm{~m}$ coherence can only be found for frequencies up to about 1000 Hz. However in this case no Doppler or propagation 
compensation has been done. The MSC is better on the right side of these plots. Right side represents the situation where the hydrophones are phasing the stern of the target (from where the sound is generated). The chosen bandwidth of each baseline is based on the coherence of the plot (Fig. 4).
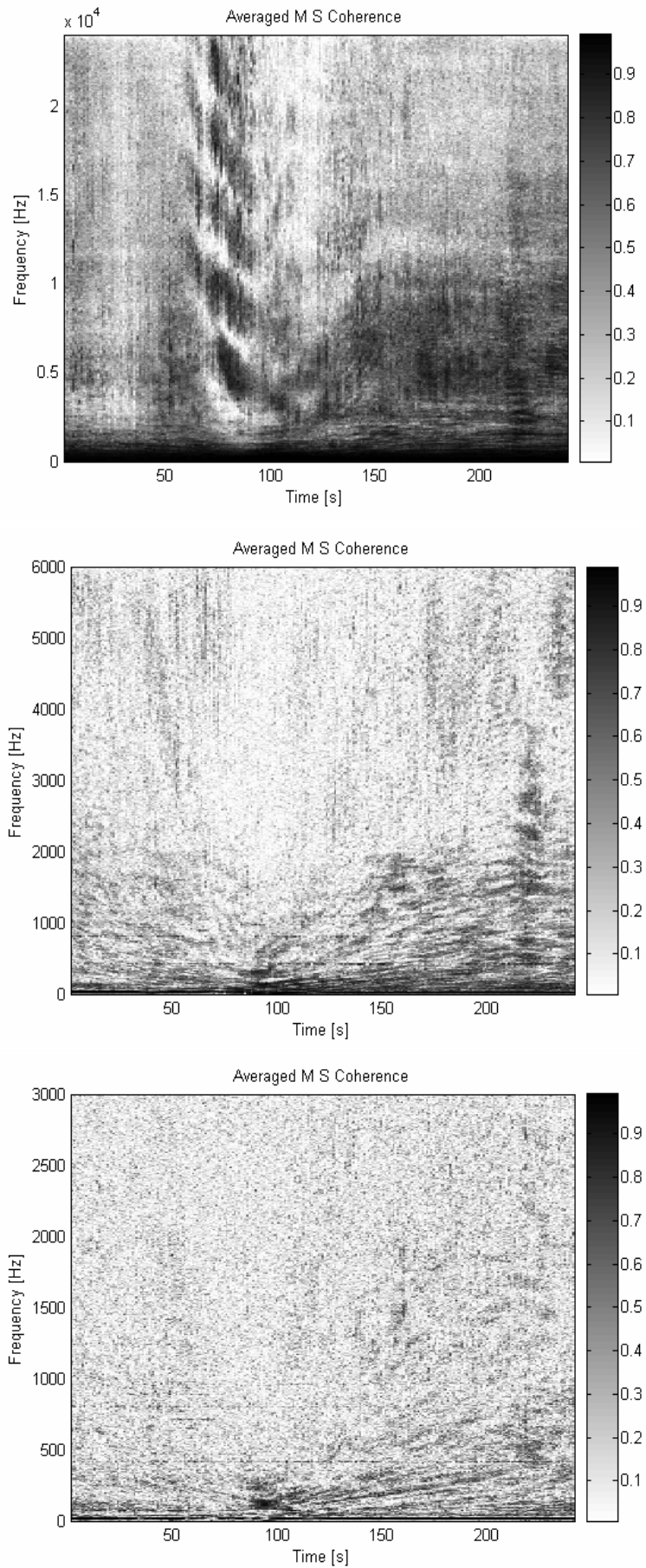

Fig. 4 Magnitude Square Coherence using Welch [10], with Hanning windowed fft's of 1024 samples, $50 \%$ overlap, analyzing time frames of 1 second totally between $x_{i}$ and $x_{j} . x_{j}$ is delayed according to the laser curves in Fig. 5. Baselines of 0.5, 9 and 30 meter is plotted, showing the shortest baseline at the top of the figure. Notice the different y-axis, the sampling frequency is 48,12 and $6 \mathrm{kHz}$ respectively.

\section{B. TDE}

Results of the TDE from the two methods are shown in Fig. 5. The lag for max of GCC-PHAT/NLMS filter coefficient is compared to the calculated time delay from laser measurement (assuming constant speed of sound with depth). We can see that the laser curve for the shortest baseline has a relatively large deviation from both estimates of the time delay. This might be due to a simple model for the spreading of sound, but more likely does it depend on incorrect assumptions about the position of the hydrophones. Small baselines are more sensitive to low accuracy of the sensors position which is a disadvantage of small apertures.
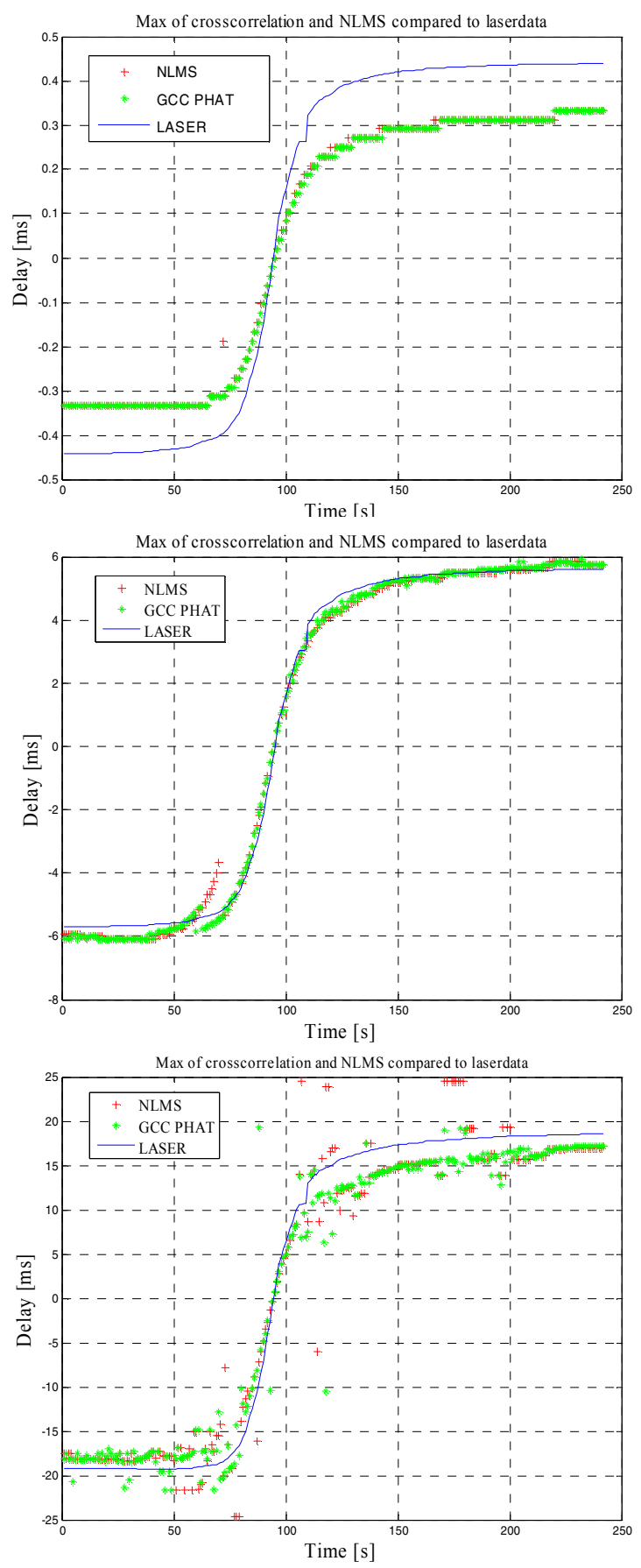

Fig. 5 Time delay estimation by two different methods compared to a calculated time delay from laser measurement for baselines of $0.5,9$ and 30 meter, showing the shortest baseline at the top of the figure. Sampling frequency is 48,12 and $6 \mathrm{kHz}$ respectively Integration time is 1 second. NLMS parameter; $\beta=0.015,0.0030,0.0034$ and length of filter $=68,128$, 296 and number of laps within the same second $=1,5,20$ respectively. 
Fig. 6 show a moment in time for the GCC-PHAT result/LMS filter coefficients. We can see a decreasing quality of the TDE (hight of max relatively the other heights) with increasing values of the baseline, which MSC in Fig. 5 also substantiate. This can depend on different traveling paths for the sound from the target to each sensor, and/or the different Doppler shift each sensor see when the baseline is long.
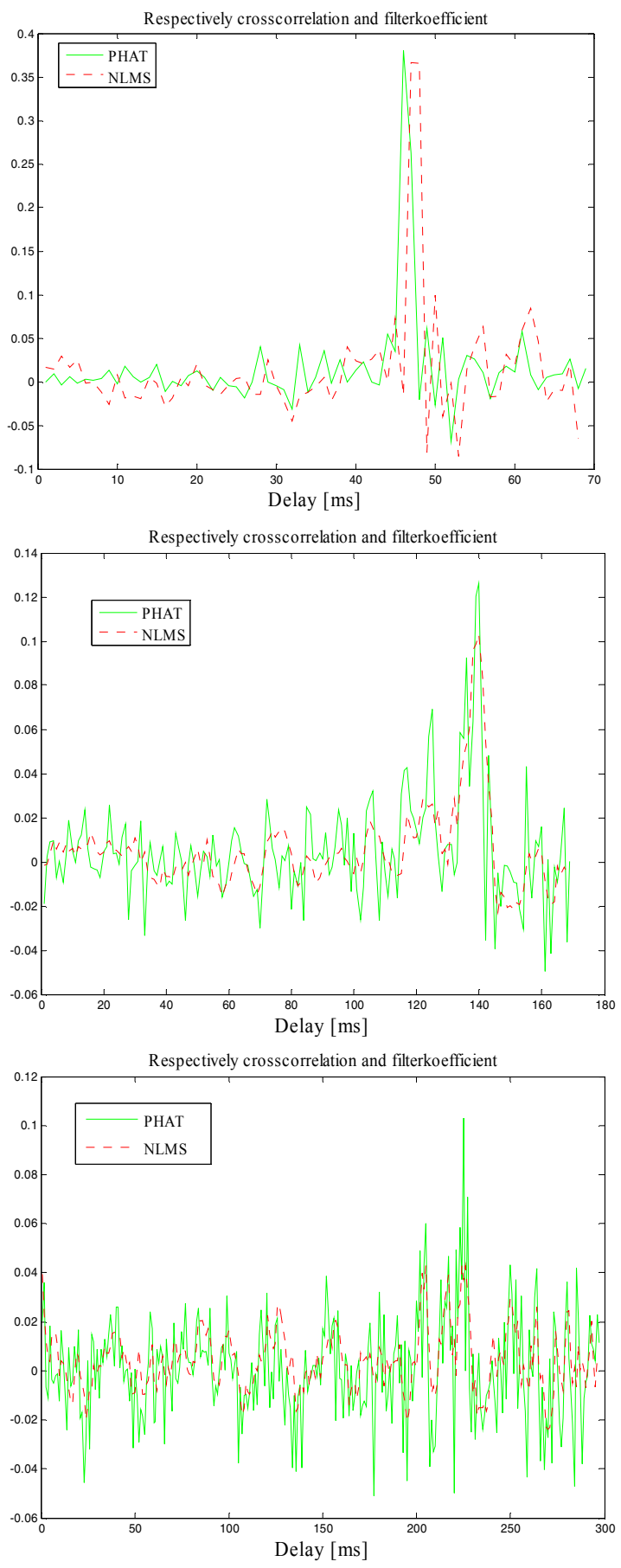

Fig. 6 Correlation and filter coefficient for time frame 130 in Fig. 5. One plot for each baseline $(0.5,9$ and $30 \mathrm{~m})$, showing the shortest baseline at the top of the figure.
The difference or error between estimated time delay and the delay calculated from the laser measurement is plotted in Fig. 7. It is however hard to state accurate facts regarding the errors, since the target it self is $10 \times 20$ meter and the hydrophone positions are not perfectly known, but this will give a rough estimate for comparison.
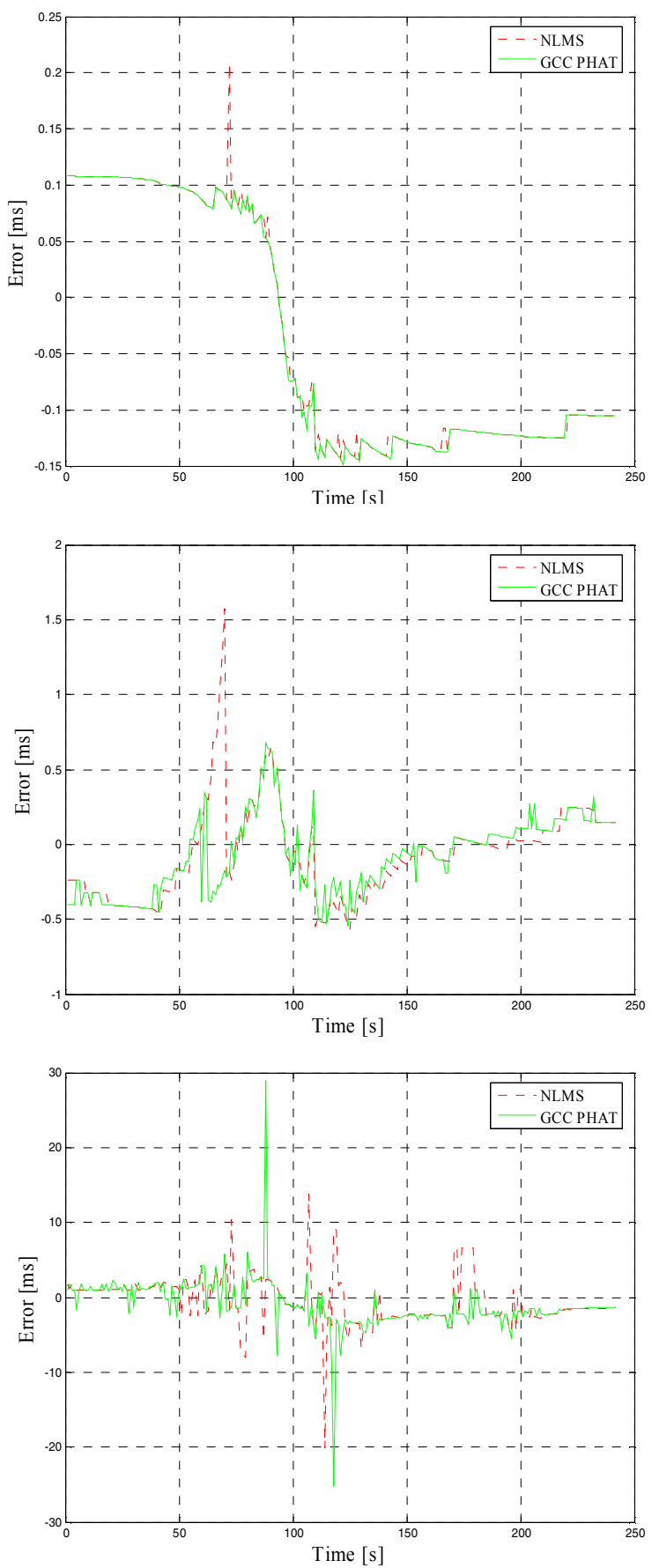

Fig. 7 The difference or error between estimated time delay and the delay calculated from the laser measurement, see Fig. 5. One plot for each baseline $(0.5,9$ and $30 \mathrm{~m})$, showing the shortest baseline at the top of the figure. 


\section{DISCUSSION}

Fig. 5 and 7 shows that the TDE from the two methods is approximately the same for the two smaller baselines, at $d=30 \mathrm{~m}$ the GCC PHAT method gives a better estimate for this target. This is also verified by the RMS value of the errors in Fig.7, given in Table 1

\begin{tabular}{rrr}
\multicolumn{4}{c}{ TABLE I } \\
RMS error \\
\hline$d$ & Result & \\
\hline & GCC-PHAT Error $_{\text {RMS }}[\mathrm{ms}]$ & NLMS Error $_{\text {RMS }}[\mathrm{ms}]$ \\
\hline 0.5 & 0,112 & 0,113 \\
\hline 9 & 0,265 & 0,333 \\
\hline 30 & 2,28 & 4,76
\end{tabular}

\section{TDE errors affect on the position estimate}

From the MSC in Fig. 4 we see a decrease in coherence as the baseline between the hydrophones increase. This is also indicated in Fig. 6 where the correlation gets weaker for larger $d$. The coherence drop for larger baselines, especially at higher frequencies, causes a larger uncertainty in the TDE compared to the laser measurements, clearly seen in Fig. 7 and Table 1. However our main interest is not the TDE error but rather the positioning error. Given a TDE the target can be anywhere on a hyperbola. The positioning error is given by the position on this specific hyperbola, and the positioning error increases as the distance to the baseline increases. This means that even though the TDE is verse for a large baseline compared to a small one the positioning accuracy can be much better. We will therefore compare the positioning error. In our case the three pairs of sensors have their baseline approximately in the same direction as the moving target velocity vector, see Fig. 3. Knowing the perpendicular distance to the baseline we can estimate the positioning error, Error $_{\text {pos }}$, in the direction of the moving target, given the TDE error, here Error $_{\text {RMS }}$. On the perpendicular axes to the baseline, when the distance, $R$, is much bigger than the baseline, the error in the moving target direction will approximately be given as

$$
\text { Error }_{\mathrm{pos}}=\mathrm{D}_{\mathrm{rel}} * \text { Error }_{\mathrm{RMS}} *_{\mathrm{c}}
$$

where $\mathrm{c}$ is speed of sound and $\mathrm{D}_{\mathrm{rel}}=R / d$ Using this approximation we can compare the three baselines and the comparison is given in Table 2.

TABLE 2

Position error

\begin{tabular}{rrrr}
\hline \multicolumn{5}{c}{ R } & \multicolumn{3}{c}{ Result } \\
\hline & Error $_{\text {RMS }}[\mathrm{ms}]$ & $\mathrm{D}_{\text {rel }}[\mathrm{R}=75 \mathrm{~m}]$ & Error $_{\text {pos }}[\mathrm{m}]$ \\
\hline 0.5 & 0,11 & 150 & 24 \\
\hline 9 & 0,265 & 8,4 & 3,2 \\
\hline 30 & 2,28 & 2,5 & 8,2
\end{tabular}

It is clearly seen from the table that we have higher position accuracy for large apertures.
The results of this study indicate a decreasing quality of the TDE with an increasing baseline, due to a decreased coherence. However, despite the fact of decreased coherence, the study indicates smaller positioning errors for the two larger baselines compared to the small one. This is of interest since we, in this work, have not been compensating for the Doppler shifts between the sensors connected to the large baseline and nor for the spreading of sound which gets more complicated for large baselines. In [1] Doppler compensation clearly improved the TDE and this has to be considered in future work on scattered arrays. Methods that consider the spreading of sound, such as Matched Field Processing, should be investigated.

In this study we only compared the TDE error for three pairs of sensors. In the experiment 8 sensors were used presenting the possibility to get the position from $7+6+5+4+3+2+1=28$ pairs of sensors. By triangulation of the different measurement pairs an accurate positioning in three dimensions can probably be achieved. Tracking algorithms such as Kalman, in combination with methods for optimum sensor combination, is a motivating topic for future work.

Another interesting aspect of scattered arrays with large baselines is the possibility for accurate positioning using only relatively low frequencies. This would provide lower sampling frequencies and therefore require less processing power. Such traits are of great importance when it comes to realization of real time systems.

\section{CONCLUSIONS}

In this paper we show results from a scattered array positioning of a moving target. Three different sensor pairs were used for passive positioning. The baseline of these three pairs was $0.5 \mathrm{~m}, 9 \mathrm{~m}$ and $30 \mathrm{~m}$, and the closest point to target was approximately 75 meters. The study indicates that scattered arrays can be used for moving target positioning with satisfying results. It was found, for this target, that the positioning errors for the two larger baselines were much smaller than for the short baseline. This despite the fact that the TDE error in time, compared to laser measurements, is bigger for the large baseline due to the decreased coherence.

It was also found that whitened generalized cross correlation and adaptive filter for time delay estimation gave the same result for the two smaller baselines. For the largest baseline the cross correlation function performed better. Investigation of more targets needs to be done in order to decide the best suited method for the application of scattered arrays.

\section{ACKNOWLEDGMENTS}

The authors would like to thank everyone at Amlab Elektronik $\mathrm{AB}$ for providing measurement data and especially Erik Asplund for his contribution to this work. 


\section{REFERENCES}

[1] B. Xerri, J.F. Cavassilas, B. Borloz., "Passive tracking in underwater acoustic" Elsevier Signal processing, vol. 82, pp. 1067-1085, 2002.

[2] M. Brandstein and D. Ward, Microphone Arrays: Signal Processing Techniques and Applications, Springer, 2001

[3] G. C. Carter, E. R. Robinson, "Ocean effects on time delay estimation requiring adaptation" IEEE Journal of oceanic engineering, vol. 18, No. 4, pp. 367-378, October 1993.

[4] P. C. Ching, H. C. So, "Two adaptive algoritms for multipath time delay estimation" IEEE Journal of oceanic engineering, vol. 19, No. 3, pp. 458-463, July 1994.

[5] Y.T. Chan; P.C. Ching, "Non-stationary time delay estimation with a multipath" Proc. ICASSP '89, vol. 4, pp. 2736-2739, May 1989.

[6] M. H. Hayes, Statistical digital signal processing and modeling, Canada: John Wiley \& Sons Inc., 1996

[7] G. C. Carter, Coherence and Time Delay Estimation: An Applied Tutorial for Research, Development, Test, and Evaluation Engineers, NY: IEEE PRESS, April 1993.

[8] C. H. Knapp, G. C. Carter, "The generalized correlation method for estimation of time delay" IEEE Trans. ASSP, vol. 24, No. 4, pp. 320-327, August 1976.

[9] J. Krolik, M. Joy, S. Pasupathy, M. Eizenman, “A comparative study of the LMS adaptive filter versus generalized cross correlation methods for time delay estimation" Proc. ICASSP '84, vol. 1, pp. 15.11/1-4, March 1984.

[10] Matlab, version 7.0.0.19920 (R14) 